Review Article

\title{
COVID-19 and Cancer Therapy: Interrelationships and Management of Cancer Cases in the Era of COVID-19
}

\author{
Simon N. Mbugua $\mathbb{D}^{1}{ }^{1}$ Lydia W. Njenga, ${ }^{1}$ Ruth A. Odhiambo $\mathbb{D},{ }^{1}$ Shem O. Wandiga, ${ }^{1}$ \\ and Martin O. Onani ${ }^{2}$ \\ ${ }^{1}$ Department of Chemistry, University of Nairobi, P.O. Box 30197-00100, Nairobi, Kenya \\ ${ }^{2}$ Department of Chemical Sciences, University of the Western Cape, Private Bag X17, Belville, Cape Town 7535, South Africa \\ Correspondence should be addressed to Simon N. Mbugua; mbuguasn77@yahoo.com
}

Received 27 March 2021; Revised 15 August 2021; Accepted 7 September 2021; Published 24 September 2021

Academic Editor: Nenad Ignjatovic

Copyright (C) 2021 Simon N. Mbugua et al. This is an open access article distributed under the Creative Commons Attribution License, which permits unrestricted use, distribution, and reproduction in any medium, provided the original work is properly cited.

\begin{abstract}
The COVID-19 global epidemic poses this generation's biggest worldwide public health challenge probably since the 1918 influenza epidemic. Recent reports on two new variants have triggered a dramatic upsurge in research to understand the pandemic, primarily focussing on the virology, triggers, clinical characteristics, and diagnostic tests including the prevention and management of the novel coronavirus. Whilst such studies are important in managing the present medical emergency, there is a need for further work to include interdependencies between the epidemic and other illnesses. This will help in developing effective approaches to treat and manage associated diseases in both the short and the long term. In this regard, people living with cancer are a subgroup that is highly vulnerable to respiratory infections and acute pneumonitis similar to the one caused by the COVID19 virus. This is because the state of their immunity is compromised due to malignancy and the adverse effects of anticancer treatments. With annual cancer projections rising globally and an estimated 70 percent of all cancer-related deaths occurring in low- and middle-income countries, the patient population with impaired immune systems that could be adversely impacted by COVID-19 is only anticipated to rise. In this review, we delve into the challenges and health risks facing cancer patients and cancer treatment in the COVID-19 context, with suggestions into viable measures which can be taken to minimize exposure to the risk of contracting COVID-19 for this vulnerable subgroup. New mutations and the prospects offered by vaccines development and how they relate to this class of patients are also discussed.
\end{abstract}

\section{Introduction}

There has been a recent report of mutated variants of the SARS-CoV-2, the virus responsible for causing COVID-19 [1]. Since the long-term effects of the virus still remain largely unknown, this has aroused concern and anxiety on the possible impacts of these viral changes. Not long ago, reports of two different SARS-CoV-2 variants emerged from the WHO authorities and they were detected in the United Kingdom and Northern Ireland. They were named VOC 202012/01, and the other, a variant from South Africa, referred to as 501Y.V2 [2]. Although no change in disease severity has been reported for the VOC 202012/01 variant, there are reports of increased transmissibility compared to
SARS-CoV-2 [2]. This further complicates the already complicated situation in that it will require further research to understand the new variants, yet the year-old SARS-CoV2 is still under study.

The new year 2021 came with high expectations that the vaccination campaigns taking place worldwide will arrest the current epidemiological situation arising from the second wave of the virus. However, since high numbers of new cases and deaths continue to hit new records, this calls for even stricter adherence to safety measures to reduce infections and deaths. Unfortunately, at the pace in which the vaccine is being procured and distributed by the most affected countries, there is a likelihood of $3^{\text {rd }}, 4^{\text {th }}$, or even $5^{\text {th }}$ waves occurring. 
The COVID-19 outbreak was formally declared a public health problem of global significance by the World Health Organization (WHO) on 30 January 2020 [3]. The advent of the SARS-CoV-2 virus responsible for the COVID-19 after the 2002 extreme acute respiratory coronavirus syndrome (SARS-CoV) and the 2012 Middle East respiratory coronavirus syndrome (MERS-CoV) represented the third advent in the twenty-first century of a remarkably infectious and large-scale coronavirus outbreak into modern civilization [4-7].

A few studies have indicated that COVID-19 mortality in elderly people and in situations with other significant comorbidities such as cancer is relatively higher [8-11]. Cancer patients, especially those with cancer of the lungs, pose a higher risk of COVID-19-related respiratory disorders [12-14]. Furthermore, the application of chemotherapeutic agents may have a detrimental effect on the patients' immunity, and therefore, it will be prudent to consider the systemic anticancer treatment (SACT) risks associated with COVID-19 [15, 16]. This risk may be determined by a number of variables such as age as well as the frequency of hospital follow-up visits which raise the risk of exposure [14]. Epidemiology statistics collected from China established that patients of advanced age were more severely impacted by the disease [17].

Prior to December 2019, six strains of coronaviruses were known to infect humans. They included two extremely infectious types, SARS-CoV and Middle East coronavirus respiratory syndrome (MERS-CoV), causing SARS and MERS, respectively [7, 8, 11, 18]. Four more types of coronaviruses involving pulmonary diseases varying from auto-resolving cold to extreme SARS-CoV pneumonia originated in China in 2002 triggering an outbreak that affected over 8,000 people with nearly 800 deaths $[9,19]$. MERS-CoV eventually appeared in the Middle East, triggering a recurrent outbreak between 2012 and 2019; however, it affected fewer people, about 2,500 people with less than 1000 fatalities [3, 20-22].

As with other coronaviruses, COVID-19 predominantly triggers infection in the respiratory system. While COVID-19 fatalities are fewer compared to those for patients infected with SARS-CoV or MERS-CoV (Singhal, 2020), COVID-19 is more transmittable, with every new case estimated to generate an average of 2.7 new secondary cases. In the COVID-19 epidemic, patients with cancer are deemed a particularly susceptible category [23, 24]. Owing to the COVID-19 pandemic, the healthcare system faces the challenge of reorganization, for not only managing the [1] pandemic effectively but also simultaneously tracking other diseases that require sustained follow-ups on treatment [25].

Reports have indicated that postponements and disruptions are related to disease development and augmented mortality among patients with thoracic, gynaecological, and head-and-neck cancers [26]. This requires an optimum balance on treatment and deferrals to mitigate COVID-19 exposure $[26,27]$. With these in mind, it has been suggested that clinical decisions should be deliberated on a case-by- case basis to ensure that the benefit gained from sustained cancer treatments are more than the hazards of contracting COVID-19 [28].

While COVID-19 in the population at large is known to have a fairly low mortality rate of 2 to 3 percent [29], cancer patients suffering from COVID-19 not only have a nearly 3fold rise in mortality rates compared to COVID-19 patients without cancer but also appear to have significantly higher adverse effects once affected by the disease [19]. Cancer patients have also a 10 -fold greater risk of nosocomial COVID-19 attacks compared to cancer-free ones [30, 31]. All in all, these results indicate that cancer patients in the current COVID-19 pandemic era are a far more vulnerable subgroup.

Towards the end of the year 2020 and in the beginning of 2021, the COVID-19 virus gave the world another shock through the discovery of new mutation strains. Towards the end of September 2020, a variant called B.1.1.7 with a large number of mutations was detected and identified in the United Kingdom which transmits easily and more rapidly and is suspected to carry a higher risk of death [2]. The same variant was identified in the United States in December of the same year [32]. Another variant called B.1.351 emerged in South Africa in October 2020 and the same variant was reported in the US at the end of January 2021 [33]. In Brazil, another variant known as P.1 was detected in early January 2021 and in the US towards the end of January 2021 [34].

Because these variants are found to spread more rapidly, this may lead to increased cases of COVID-19 which will definitely add more stress on healthcare systems, leading to increased hospital visits and admissions and possibly a greater number of deaths.

1.1. SARS-CoV-2: Virology and Drug Targets. Unlike the SARS-CoV and the MERS-CoV, most SARS-CoV-2 fatalities shocked the medical fraternity by triggering multiorgan failure syndrome instead of a respiratory dysfunction [35]. This may be attributed to the widespread proliferation of angiotensin-converting enzyme 2 (ACE-2) which is the functioning receptor for SARS-CoV-2 into various organs [36-38]. The virus is a single-stranded RNA encased strain which targets cells via structural protein spikes (S protein) that attaches themselves to the angiotensin-converting enzyme 2 (ACE2) receptor [37, 39], as indicated in Figures 1(a) and $1(\mathrm{~b})$.

The virus then uses these host-cell receptor binding sites and endosomes to enter cells [38]. The viral polyproteins that express the replicase-transcriptase complex are synthesized once the virus enters the cell $[41,42]$. The virus produces RNA using its RNA-dependent polymerase. Functional proteins are synthesized, culminating in viral particles assembly and final release $[43,44]$. These stages in the life cycle of the virus may offer possible drug treatment targets.

Possible targets include nonstructural proteins having similar homology to other novel coronaviruses [45-47]. Other targets involve pathways for viral entry and immune modulation. 


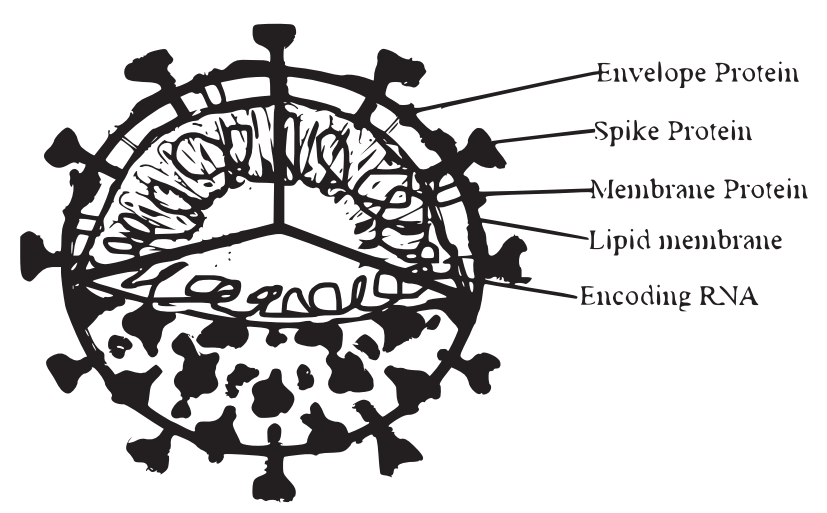

(a)
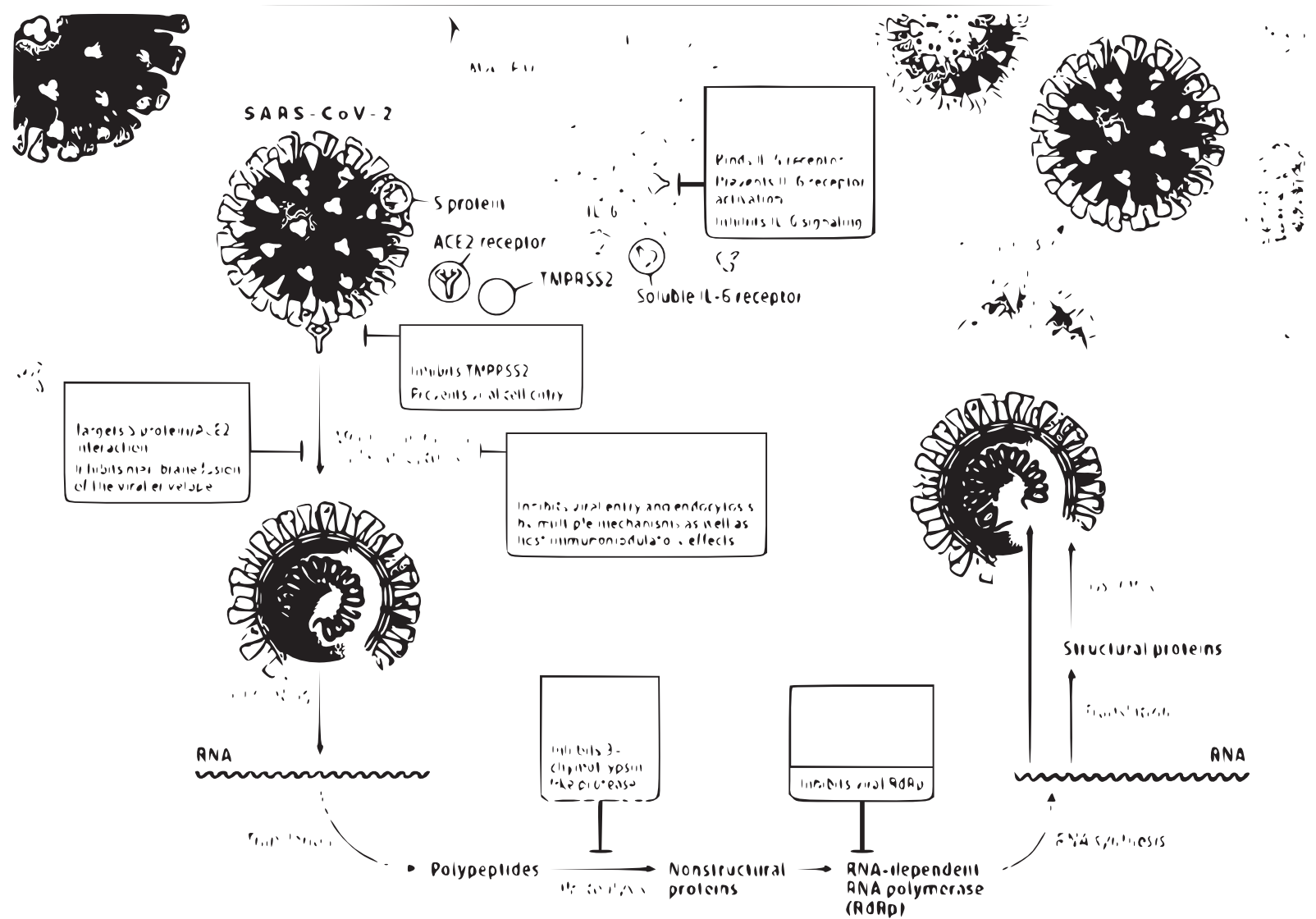

(b)

Figure 1: (a) Structure of SARS-CoV-2 and (b) virology and drug targets: viral lifecycle and potential drug targets (adapted from [40]).

Studies have shown that the magnitude of patients infected with SARS-CoV-2 virus is significantly influenced by the type of cancer [48, 49]. Patients with haematological cancer have the highest degree of severity and death across all cancer patients followed by lung cancer $[12,50]$. Of concern, too, is the impact that coronavirus epidemic might have on the medication supplies as the epidemic disrupts the pharmaceutical production and distribution processes in China, the second-largest exporter of drugs and biologics $[51,52]$.

Haematological cancer patients including patients with leukaemia, myeloma, and lymphoma are expected to have a more weakened immunity than patients with solid tumours where the cancer is localized $[48,53]$. These patients have been shown to have a rapidly deteriorating clinical course once infected with COVID-19. This is due to the fact that cancerous or ineffective immune cells, lymphocytes, or white blood cells generally cannot function optimally leading to a much-lowered immunity in haematological malignancies, leading to more severe outcomes. The total lymphocyte count and neutrophil-to-lymphocyte ratio (NLR) have been evaluated and can be regarded as one of the indicators of the status of immunity levels since lymphocytes are necessary for defence and protection against viruses and cancers [11]. 
Both lymphocyte levels and the NLR have been shown to be significant prognostic factors in patients with cancers or virus infections [18].

Figure 2 shows that the most prevalent cancer is lung cancer. This has a direct relationship with COVID-19 which is a respiratory disease affecting the lungs.

As shown in Figure 3, with an estimated rise in lung cancer as years go by, respiratory diseases will become more and more significant requiring thorough studies into the comanagement for the diseases.

The stage of cancer treatment plays an important role in mortality and morbidity risk for COVID-19 patients. The type of treatment administered influences the enormity and fatality in cancer patients once they contract COVID-19 $[11,54]$. In the recent past, immunotherapy has taken on a very significant role in the treatment of tumours by interfering with cancer cells' immune-escape routes [55]. Yet new research has shown that immunotherapy patients have the greatest mortality risk and the greatest seriousness of illness compared to cancer patients treated with other therapies, a somewhat surprising result $[53,56]$. Acute respiratory distress syndrome (ARDS) caused by cytokine storm is reported to be the major cause of death of COVID-19-infected patients, according to pathological reports $[3,57,58]$. In this context, immunotherapy may induce the production of large quantities of cytokines, which could be noxious to healthy cells, which include lung epithelial cells, thus giving rise to a more serious illness. Both postoperative cancer patients and those with developed lung cancer have been shown to have lower lymphocyte counts than healthy people [59]. In immune-compromised patients, unchecked virus infection can trigger significant cell damage and induce $\mathrm{T}$-cell fatigue and even cytokine storms which are typical causes for critical conditions [60].

COVID-19 patients with both active treatment and cancer history have a higher risk of developing serious complications compared to noncancerous COVID-19 patients [61]. This suggests that the immunity of cancer survivors remains compromised over a period of time. In comparison, patients who have undergone chemotherapy or similar operation in the near past have a statistically greater chance of developing medically serious complications (75 percent) unlike those who have not undertaken these procedures [62].

\section{Methodology}

The main objective of this review was to highlight the challenges of cancer care in the era of COVID-19 and the possible mitigations in form of practical suggestions which can be implemented to protect cancer patients from exposure to the SARS-CoV-2. To achieve this, a scoping analysis was undertaken using the following five steps:

(i) Identifying a clear research objective and search strategies

(ii) Searching the relevant scholarly publications

(iii) Screening of the articles that fitted our objectives

(iv) Extracting data (v) Analysing, discussing, drawing conclusions, and reporting the results

A thorough online review of the literature was conducted for relevant publications from December 2019 to December 2020. The keywords "cancer" or "oncology" were used in combination with "covid-19," and/or "SARS-CoV-2." The search was conducted in Google Scholar, Google search, PubMed bioRxiv, medRxiv, and ChemRxiv. A total of 126 publications were retrieved. Relevance was determined by the use of Preferred Reporting Items for Systematic Reviews and Meta-Analyses (PRISMA) flowchart. Selected articles of the recovered publications were reviewed for relevance where texts were examined and 94 suitable papers that tarried well with the objectives of the review were selected. An analysis of cited references appearing in some of the selected papers was also carried out.

The methodology is summarized in the PRISMA flowchart using the layout appearing in the PRISMA website as shown in Figure 4.

2.1. COVID-19 and Cancer Management/Care. Studies conducted in China showed that cancer patients, mainly those who lately had received chemotherapy or surgery, were at greater risk of negative consequences subsequent to SARS-CoV-2 infection. Investigations of 3,920 patients pointed out the greatest mortality in patients who had undergone treatment in the preceding 1-3 months. Ratios were maximum in those who had undergone chemo- or immunotherapy in a span of 2 weeks of SARS-CoV-2 cases. The International Severe Acute Respiratory and Emerging Infections Consortium, (ISARIC)-4C COVID-19 Clinical Information Network (CO-CIN), based in the United Kingdom, is a collaborative data collection network from hospital in-patients with confirmed or clinically probable SARS-CoV-2 cases. Data obtained from 20,133 participants already hospitalised from SARS-CoV-2 infections proposed that cancer was a major issue linked to SARS-CoV-2 deaths. During the initial studies on cancer-specific cases, 1,797 (8.6\%) of the patients were found to have malignant neoplasm, with a mortality rate of $35 \%$. Later updated data pointed out cancer as a risk issue for mortality in all age groups, with a mortality rate of $40.5 \%$. The study included participants from over 70 centers across the United Kingdom and surveyed 800 participants with cancer having received treatment in a span of 12 months. The study reported a mortality rate of $28 \%$ in a population of symptomatic patients in secondary care. This early analysis reported a $76 \%$ ratio of patients who were on active treatment 4 weeks prior to SARS-CoV-2 confirmation and identified age, male gender, and comorbidities as risk factors for mortality. A study on 351 patients by Dutch Oncology and COVID-19 consortium on solid organ and haematological malignancies, with serological or radiological confirmation of SARS-CoV-2 infection, reported a rate of $32.3 \%$, with age $>65$ years, male gender, previous/active haematological or lung cancer cases pointed out as risk factors of mortality. 
Most Common Cancers 2018

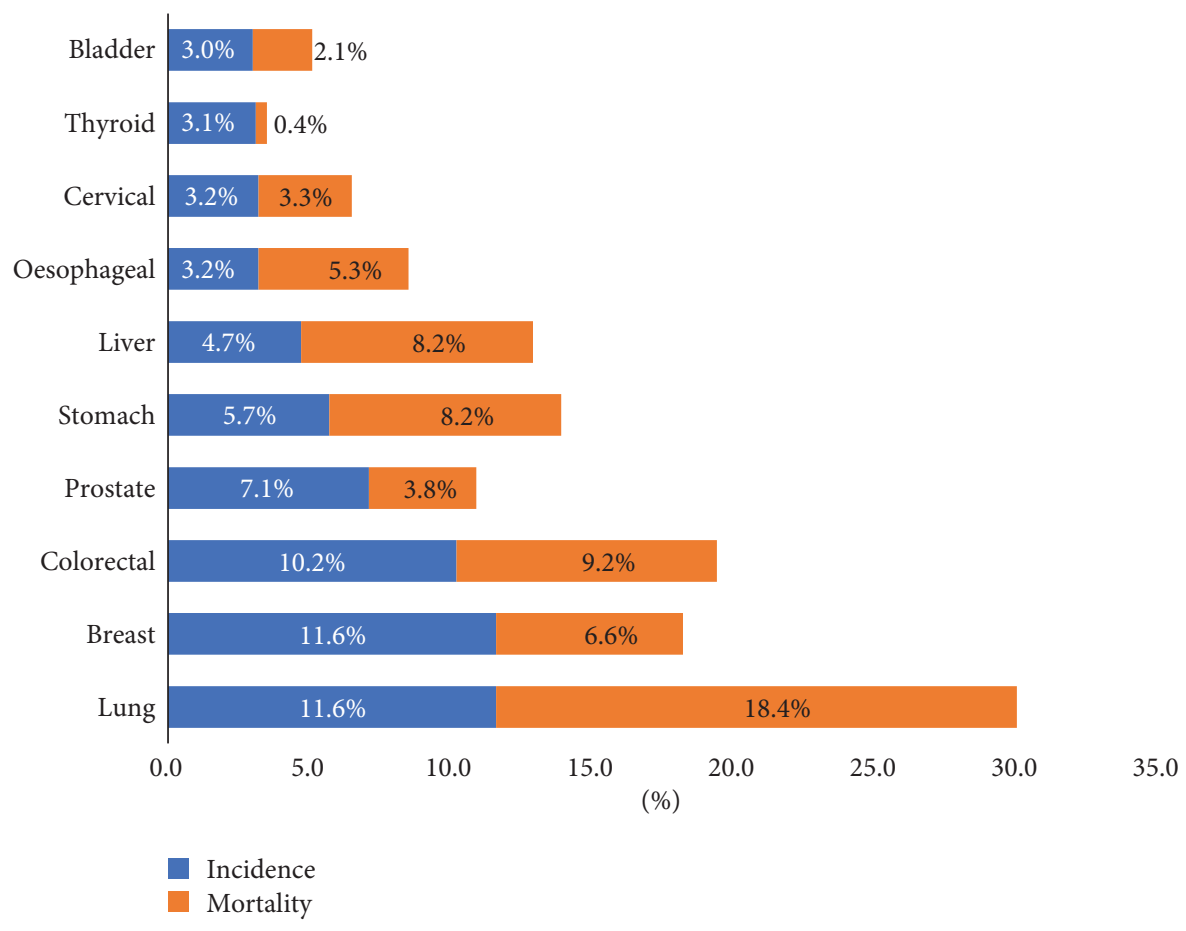

Figure 2: Global cancer burden as of 2018 (WHO 2020).

Estimated Trends in Breast and Lung Cancers

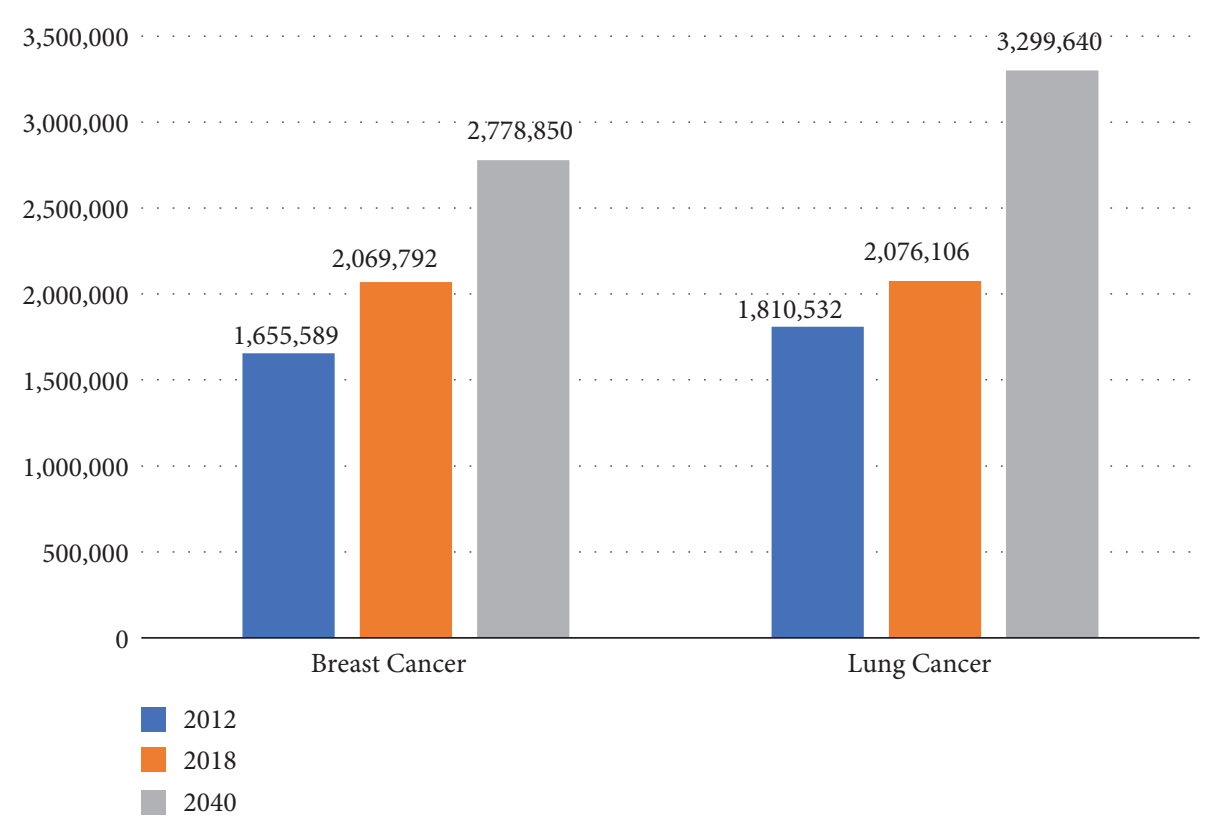

Figure 3: Estimated past and future trends in total cases per year (breast and lung).

Another investigation carried out in 2020 on 357 participants from 23 Swiss centers showed that $57 \%$ of the participants who had undergone anticancer therapy in a span of 90 days subsequent to being diagnosed with SARSCoV-2 had a mortality rate of $18 \%$. This study correlated age and cancer treatment to worse outcomes.
In 2018, cancer was accountable for approximately 9.6 million fatalities and is estimated to be liable for 1 in 6 fatalities worldwide in 2020 [63]. Cancer patients are more vulnerable to illness than cancer-free people owing to a chronic immunosuppressive condition triggered by metastasis and cancer therapy agents, such as chemotherapy or 


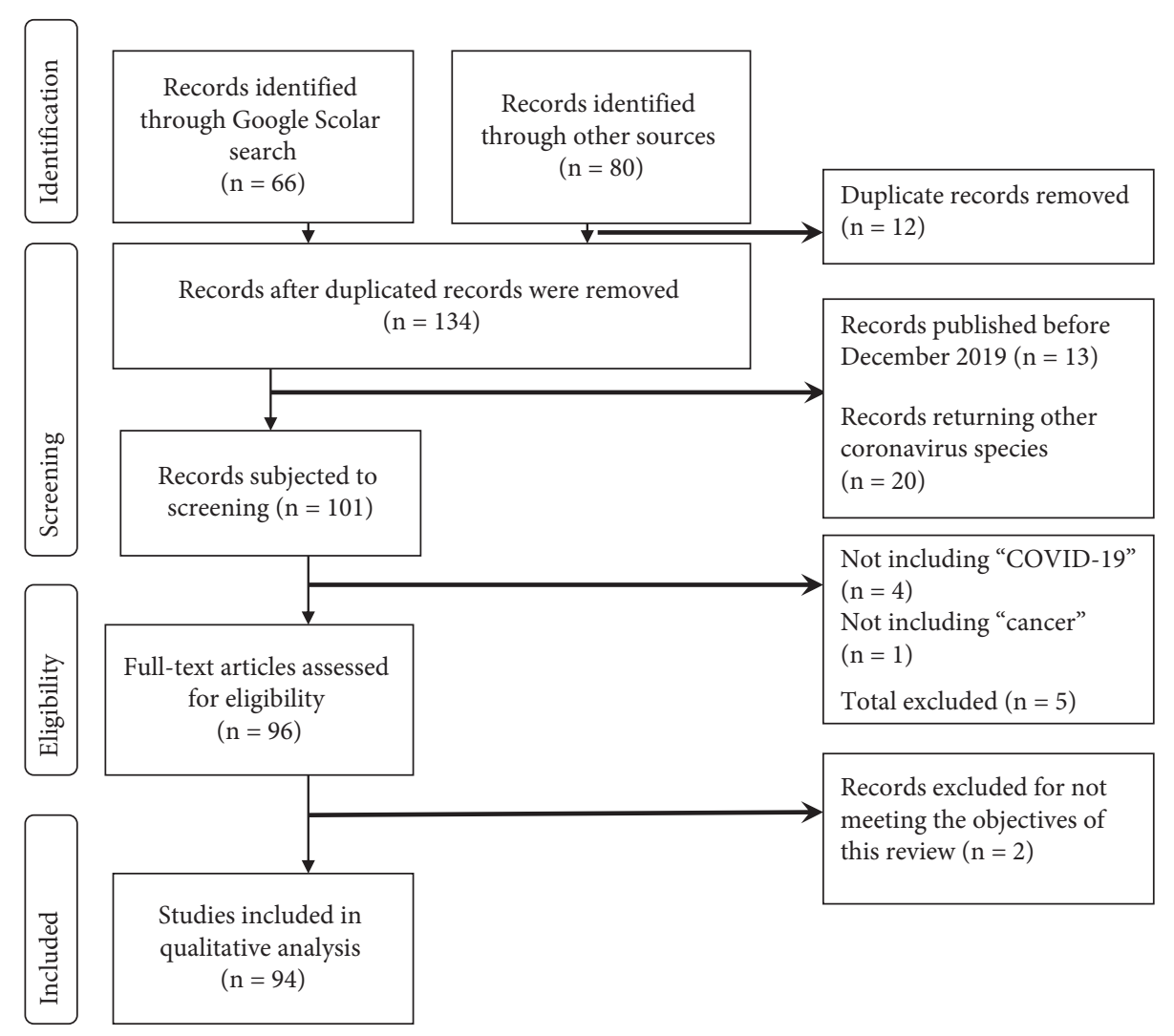

FIgURe 4: PRISMA flowchart used for the methodology.

surgical procedures $[49,53,62,63]$. According to a recently released Chinese study, the chance for patients with cancer to experience extreme outcomes in terms of critical care admission, intrusive breathing, or death, relative to people without cancer, was 39 percent versus 8 percent $[3,61]$. The age factor plays out in that most cancers occur at a later age affecting the older population to a larger extent, further compromising this age group with an already compromised immunity. Reports indicate that, in the United States, 80 percent of all cases are found in people 65 years of age and above, with similar observations made in studies conducted in Wuhan, China [64].

COVID-19 pandemic has posed a major challenge in almost every hospital especially in areas requiring ICU support, particularly the surgical units and others requiring respiratory aiding equipment. The presence of COVID-19infected patients in the ICU typically creates an extra burden which limits the capacity of non-COVID-19 patients in the ICU to receive optimum treatment [65].

Hospital admissions and repeated hospital visits have been classified as possible hazard factors for the infection of COVID-19 [66]. Moreover, while research concludes that cancer patients have worse COVID-19 outcomes, it also indicates that the mean age of such patients (63.1 years) is slightly higher compared to those without cancer (48.7 years), indicating that advanced age was correlated with worse COVID-19 results $[9,11,62,66]$.

The World Health Organization (WHO) declared COVID-19 an international public health crisis and raised its risk assessment from "high" to "very high" with a similar declaration made in Korea $[63,67,68]$. Taking into account that there are high and still rising numbers of infected persons with SARS-CoV-2, cancer should not be ignored as a coexisting illness. This is due to the fact that cancer patients are frequently recalled to hospitals for treatment and disease monitoring, and medical procedures such as chemotherapy or radiation therapy are immune suppressants, raising the risk of contracting COVID-19. Similarly, the risks faced by cancer patients should equally be raised to very high.

\subsection{COVID-19 Vaccines and Cancer Patients. COVID-19} vaccines currently in use are anticipated to offer protection against the virus and the new variants [69]. These vaccines have been designed to provoke a wide-ranging immune response specifically invoking the cells to produce a harmless copy of a virus's spike protein, and, therefore, the mutated variants will not render the vaccines ineffective [70].

Wide-scale production and distribution of vaccines still remains the most critical way of fighting the variants and protecting people who might be exposed. This in turn reduces the spread rates and might be effective in the long run in the elimination of the virus and its variants [71]. The current priority of vaccination for "high-risk" groups like doctors and frontline caregivers is also a good move in minimizing the chances of transmission and spread [72]. The more the number of people who access the vaccines, the less the transmission, and this will result in fewer mutations 
[71]. While this is the case, we still need to take all precautions to arrest the spreading of the virus and thereby avert possible mutations that may diminish the effectiveness of present vaccines.

For cancer patients, the key worry about the vaccines is not their safety, but about how effective they will be considering the weakened immunity for people with cancer [59]. As discussed above, treatments like chemotherapy, radiotherapy, or immunotherapy affect the immune system which may compromise the efficacy of the vaccines [73]. However, most conclusions on the effects of COVID-19 vaccines on cancer patients are subjective because initial tests with these vaccines did not include people already undergoing treatment with immunosuppressant drugs like chemotherapy drugs, or people with already weakened immune systems. The initial focus was on people with strong and vibrant immune systems [4]. Therefore, there is no conclusive data on the efficacy of these vaccines for these groups of people.

The lack of conclusive information does not imply that these vaccines will not work on this class of people. We might however expect that they might not be as effective in people with weakened immune systems as in people with vibrant immune systems [74]. Because of the higher risk this class of people face in terms of being more adversely affected by the COVID-19 variants, it will be best for them to get vaccinated [59]. It can be concluded that getting some protection from the vaccine is better than not having any protection [59].

As with many new vaccines, there are health concerns raised not only on efficacy but on adverse side effects [75]. These should, however, not make us suspend the exercise of getting vaccinated. We need to utilize the tools available even as research continues to make improvements.

\section{Results and Discussion}

Out of the 94 publications reviewed, 90 were journal articles and 4 were cohort studies involving retrospective case studies and cross-sectional analyses. All the reviewed articles were only selected for either the relevant acronyms of "COVID-19" or "SARS-CoV-2" and the terms "cancer" or "oncology."

In lieu of the COVID-19 epidemic and other future infectious outbreaks which may have direct impacts on cancer patients, deliberate postponement of adjuvant chemotherapy or surgical procedures may be considered in mild cases. Since the immunity of cancer survivors remains progressively compromised over a period of time, the relevant authorities should freely provide effective personal protective equipment for cancer victims or survivors who may need to visit hospitals [76]. When cancer patients get infected with COVID-19, more rigorous monitoring or treatment regimens should be urgently considered, particularly in the older patients and those with other comorbidities [77]. Initiatives that could make it possible to manage cancer patients at home must also be considered.

These can be achieved through telecare and video calls with the physicians to reduce hospital visits, as well as replacing invasive treatments with oral medication where practicable [78]. This will be an effective method in providing noncontact follow-ups on management and care to cancer patients [79]. Patients who find themselves in an affected environment should be immediately isolated. Resources and equipment to enable intravenous anticancer drugs may be delivered at home where applicable. Chemotherapy dosage may also be adjusted and radiotherapy hypofractionated as an additional measure to reduce hospital visits [80]. Studies conducted on radiotherapy on cervical cancer during the COVID-19 era suggest that hypofractionation may be more relevant in this era due to the experienced pressure on medicare facilities occasioned by the COVID-19 pandemic [81].

Furthermore, at the recommendation of the prescribing oncologist, certain patients with slowly developing metastatic tumours may be granted brief gaps in their care, with condition monitoring extended to prevent hospital admissions [82]. However, despite these steps, certain cancer patients may continue with hospital admission for routine treatment or radiotherapy.

In order to ensure that such patients do not show any complications consistent with COVID-19 before being admitted to oncology or radiotherapy wards, it would be prudent for caregivers to make regular calls to those scheduled for admission, just to know their status [83, 84]. The greatest threat for cancer patients in this pandemic is the difficulty or inability to obtain requisite healthcare services in terms of getting to the hospital and receiving regular follow-ups [83]. This is mainly in part due to movement restrictions and imposed isolation and quarantine advisories. Country-specific strategies will need to be adopted in the future where countries will have to reorganize the healthcare systems for both cancer treatment and other major comorbidities [85].

Decisions as to whether or not to postpone cancer therapy for people already infected with COVID-19 should be taken on a case-by-case basis [86]. This may depend on the threat to the individual and their prevailing conditions as delays may contribute to worsening of cancer and eventually to worse outcomes [87]. If admission to the hospital is considered unavoidable, admissions should be referred to the departments concerned in fighting COVID-19 so that facilities for oncology and radiation therapy remain free of COVID-19 [88]. This is because cancer wards might not be fitted with the requisite quarantine facilities necessary in COVID-19 situations. Furthermore, outpatient facilities should implement demarcation measures (e.g., minimum seating space to maintain social distance, movable walls, patient and staff wearing masks) to protect patients with cancer.

\section{Conclusion}

In conclusion, this study shows that patients with cancer and/or cancer-related complications are at an obvious higher risk of worse outcomes subsequent to SARS-CoV-2 infection in comparison to cancer-free patients, where mortality rates have been shown to range from 13 to $40.5 \%$. Factors like age and preexisting comorbidities have also been 
associated with worse outcomes in patients with cancer who acquire SARS-CoV-2.

We believe that this short but comprehensive review will definitely be of help to decision-makers, to assist in giving direction in the management of cancer patients impacted by the current COVID-19 outbreak. The same procedures are also applicable to other patients who might require constant hospital visits thereby raising their risk of contracting COVID-19. This will be most appropriate especially for African and other developing countries, whose healthcare systems not only are strained but also lack most of the intensive care facilities needed in COVID-19 situations. Adapting telecare and other home-based consultations and follow-ups will relieve our healthcare facilities of both human-to-human interactions and reduce the associated risks of patients carrying the virus home and also leave the medical personnel available to address the very critical cases.

\section{Data Availability}

All data used to support the findings of this study are included within the article.

\section{Conflicts of Interest}

The authors declare that they have no conflicts of interest.

\section{Acknowledgments}

The authors acknowledge the support of the International Science Program, ISP, under KEN-01 project for the funding and NRF, South Africa. They also acknowledge the Department of Chemistry, University of Nairobi, for support and guidance in this work, and the Department of Chemistry, University of the Western Cape (UWC), South Africa.

\section{References}

[1] A. Awadasseid, Y. Wu, Y. Tanaka, and W. Zhang, "SARS-cov2 variants evolved during the early stage of the pandemic and effects of mutations on adaptation in Wuhan populations," International Journal of Biological Sciences, vol. 17, no. 1, pp. 97-106, 2020, https://doi.org/10.7150/ijbs.47827.

[2] N. G. Davies, S. Abbott, R. C. Barnard et al., "Estimated transmissibility and impact of SARS-CoV-2 lineage B.1.1.7 in England," Science, vol. 372, no. 6538, 2021.

[3] M.-Y. Dai, D. Liu, M. Liu et al., "Abstract CT406: patients with cancer appear more vulnerable to SARS-COV-2: a multicenter study during the COVID-19 outbreak," in Proceedings: AACR Annual Meeting 2020, Philadelphia, PA, USA, April 2020, https://doi.org/10.1158/1538-7445.am2020-ct406.

[4] E. Mahase, "Coronavirus covid-19 has killed more people than SARS and MERS combined, despite lower case fatality rate," BMJ, vol. 368, 2020.

[5] J. H. Park, G. Saravanakumar, K. Kim, and I. C. Kwon, "Targeted delivery of low molecular drugs using chitosan and its derivatives," Advanced Drug Delivery Reviews, vol. 62, no. 1 , pp. $28-41,2010$.

[6] N. C. Peeri, N. Shrestha, M. Siddikur Rahman et al., "The SARS, MERS and novel coronavirus (COVID-19) epidemics, the newest and biggest global health threats: what lessons have we learned?" in International Journal of Epidemiology, vol. 49, no. 3, pp. 717-726, 2021, https://doi.org/10.1093/IJE/ DYAA033.

[7] E. Prompetchara, C. Ketloy, and T. Palaga, "Immune responses in COVID-19 and potential vaccines: lessons learned from SARS and MERS epidemic," Asian Pacific Journal of Allergy and Immunology, vol. 38, no. 1, pp. 1-9, 2020.

[8] W. Liang, W. Guan, R. Chen et al., "Cancer patients in SARSCoV-2 infection: a nationwide analysis in China," The Lancet Oncology, vol. 21, no. 3, pp. 335-337, 2020.

[9] J. Liu, Y. Liu, P. Xiang et al., "Neutrophil-to-Lymphocyte ratio predicts severe illness patients with 2019 novel coronavirus in the early stage," Journal of Translational Medicine, vol. 18, p. 206, 2020, https://doi.org/10.1101/2020.02.10.20021584.

[10] J. Yang, Y. Zheng, X. Gou et al., "Journal pre-proof prevalence of comorbidities in the novel Wuhan coronavirus (COVID19) infection: a systematic review and meta-analysis," International Journal of Infectious Diseases, vol. 94, pp. 91-95, 2020.

[11] B. Zhang, X. Zhou, C. Zhu et al., "Immune phenotyping based on neutrophil-to-lymphocyte ratio and IgG predicts disease severity and outcome for patients with COVID-19," Frontiers in Molecular Biosciences, vol. 7, 2020, https://doi.org/10.1101/ 2020.03.12.20035048.

[12] L. Bonomi, L. Ghilardi, E. Arnoldi, C. A. Tondini, and A. C. Bettini, "A rapid fatal evolution of coronavirus disease19 in a patient with advanced lung cancer with a long-time response to nivolumab," Journal of Thoracic Oncology: Official Publication of the International Association for the Study of Lung Cancer, vol. 15, no. 6, pp. e83-e85, 2020.

[13] A. Passaro, S. Peters, T. S. K. Mok, I. Attili, T. Mitsudomi, and F. de Marinis, "Testing for COVID-19 in lung cancer patients," Annals of Oncology, vol. 31, no. 7, pp. 832-834, 2020.

[14] S. Tian, W. Hu, L. Niu, H. Liu, H. Xu, and S. Y. Xiao, "Pulmonary pathology of early-phase 2019 novel coronavirus (COVID-19) pneumonia in two patients with lung cancer," Journal of Thoracic Oncology, vol. 15, no. 5, pp. 700-704, 2020.

[15] G. Banna, A. Curioni-Fontecedro, A. Friedlaender, and A. Addeo, "How we treat patients with lung cancer during the SARS-CoV-2 pandemic: primum non nocere," in ESMO Open, vol. 5, no. 2, 2020, https://doi.org/10.1136/esmoopen2020-000765.

[16] T. K. Burki, "Cancer guidelines during the COVID-19 pandemic," The Lancet Oncology, vol. 21, no. 5, pp. 629-630, 2020.

[17] I. Asokan, S. V. Rabadia, and E. H. Yang, "The COVID-19 pandemic and its impact on the cardio-oncology population," Current Oncology Reports, vol. 22, no. 6, p. 60, 2020.

[18] Y. Liu, A. A. Gayle, A. Wilder-Smith, and J. Rocklöv, "The reproductive number of COVID-19 is higher compared to SARS coronavirus," Journal of Travel Medicine, vol. 27, no. 2, 2020.

[19] L. Zhang, F. Zhu, L. Xie et al., "Clinical characteristics of COVID-19-infected cancer patients: a retrospective case study in three hospitals within Wuhan, China," Annals of Oncology, vol. 31, no. 7, pp. 894-901, 2020.

[20] P. I. Lee and P. R. Hsueh, "Emerging threats from zoonotic coronaviruses-from SARS and MERS to 2019-nCoV," Journal of Microbiology, Immunology and Infection, vol. 53, no. 3, pp. $365-367,2020$.

[21] S. A. Meo, A. M. Alhowikan, T. A. L. Khlaiwi et al., "Novel coronavirus 2019-nCoV: prevalence, biological and clinical characteristics comparison with SARS-CoV and MERS-CoV," in European Review for Medical and Pharmacological Sciences, vol. 24, no. 4, pp. 2012-2019, 2020, https://doi.org/10.26355/ eurrev_202002_20379. 
[22] C. Yeo, S. Kaushal, and D. Yeo, "Enteric involvement of coronaviruses: is faecal-oral transmission of SARS-CoV-2 possible?" The Lancet Gastroenterology and Hepatology, vol. 5, no. 4 , pp. 335-337, 2020.

[23] L. L. Lai and T. Y. Dong, "A novel method to synthesize unsymmetrical disubstituted ferrocenes," Journal of the Chemical Society, Chemical Communications, vol. 20, pp. 2347-2348, 1994.

[24] H. Wang, Z. Wang, Y. Dong et al., "Phase-adjusted estimation of the number of coronavirus disease 2019 cases in wuhan, China," Cell Discovery, vol. 6, no. 1, p. 10, 2020.

[25] J. Van de Haar, L. R. Hoes, C. E. Coles et al., "Author Correction: caring for patients with cancer in the COVID-19 era," Nature Medicine, vol. 26, no. 5, pp. 665-671, 2020 b.

[26] M. S. Ning, M. F. McAleer, M. D. Jeter et al., "Mitigating the impact of COVID-19 on oncology: clinical and operational lessons from a prospective radiation oncology cohort tested for COVID-19," Radiotherapy and Oncology, vol. 148, pp. 252-257, 2020.

[27] V. Jindal, K. K. Sahu, S. Gaikazian, A. D. Siddiqui, and I. Jaiyesimi, "Cancer treatment during COVID-19 pandemic," Medical Oncology, vol. 37, no. 7, p. 58, 2020.

[28] D. D. Lin, T. Meghal, P. Murthy et al., "Chemotherapy treatment modifications during the COVID-19 outbreak at a community cancer center in New York city," JCO Global Oncology, vol. 6, 2020.

[29] A. Aminian, S. Safari, A. Razeghian-Jahromi, M. Ghorbani, and C. P. Delaney, "COVID-19 outbreak and surgical practice: unexpected fatality in perioperative period," Annals of Surgery, vol. 272, no. 1, pp. e27-e29, 2020.

[30] P. Moss, G. Barlow, N. Easom, P. Lillie, and A. Samson, "Lessons for managing high-consequence infections from first COVID-19 cases in the UK," The Lancet, vol. 395, no. 10227, p. e46, 2020.

[31] Y. Zhang, L. Zheng, L. Liu, M. Zhao, J. Xiao, and Q. Zhao, "Liver impairment in COVID-19 patients: a retrospective analysis of 115 cases from a single centre in Wuhan city, China," Liver International, vol. 40, no. 9, pp. 2095-2103, 2020.

[32] S. E. Galloway, P. Paul, D. R. MacCannell et al., "Emergence of SARS-CoV-2 B.1.1.7 lineage," Morbidity and Mortality Weekly Report, vol. 70, no. 3, pp. 95-99, 2021.

[33] H. Wang and L. Zhang, "Risk of COVID-19 for patients with cancer," The Lancet Oncology, vol. 21, no. 4, p. e181, 2020.

[34] R. P. Walensky, H. T. Walke, and A. S. Fauci, "SARS-CoV-2 variants of concern in the United States-challenges and opportunities," JAMA-Journal of the American Medical Association, vol. 325, no. 11, pp. 1037-1038, 2021.

[35] H. K. Siddiqi and M. R. Mehra, "COVID-19 illness in native and immunosuppressed states: a clinical-therapeutic staging proposal," The Journal of Heart and Lung Transplantation, vol. 39, no. 5, pp. 405-407, 2020.

[36] T. Chen, D. Wu, H. Chen et al., "Clinical characteristics of 113 deceased patients with coronavirus disease 2019: retrospective study," BMJ, vol. 368, 2020.

[37] A. B. Patel and A. Verma, "COVID-19 and angiotensinconverting enzyme inhibitors and angiotensin receptor blockers: what is the evidence?" in JAMA-Journal of the American Medical Association, vol. 323, no. 18, pp. 1769-1770, 2020, https://doi.org/10.1001/jama.2020.4812.

[38] H. Zhang, J. M. Penninger, Y. Li, N. Zhong, and A. S. Slutsky, "Angiotensin-converting enzyme 2 (ACE2) as a SARS-CoV-2 receptor: molecular mechanisms and potential therapeutic target," Intensive Care Medicine, vol. 46, no. 4, pp. 586-590, 2020.
[39] M. A. Shereen, S. Khan, A. Kazmi, N. Bashir, and R. Siddique, "COVID-19 infection: origin, transmission, and characteristics of human coronaviruses," Journal of Advanced Research, vol. 24, pp. 91-98, 2020.

[40] J. M. Sanders, M. L. Monogue, T. Z. Jodlowski, and J. B. Cutrell, "Pharmacologic treatments for coronavirus disease 2019 (COVID-19): a review," JAMA-Journal of the American Medical Association, vol. 323, no. 18, pp. 1824-1836, 2020.

[41] R. A. Khailany, M. Safdar, and M. Ozaslan, "Genomic characterization of a novel SARS-CoV-2," Gene Reports, vol. 19, 2020.

[42] Y. A. Malik, "Properties of coronavirus and SARS-CoV-2," in Malaysian Journal of Pathology, vol. 42, no. 1, pp. 3-11, 2020.

[43] M. Dai, D. Liu, M. Liu et al., "Patients with cancer appear more vulnerable to SARS-CoV-2: a multicenter study during the COVID-19 outbreak," Cancer Discovery, vol. 10, no. 6, 2020.

[44] K. Dhama, K. Sharun, R. Tiwari et al., "COVID-19, an emerging coronavirus infection: advances and prospects in designing and developing vaccines, immunotherapeutics, and therapeutics," Human Vaccines and Immunotherapeutics, vol. 16, no. 6, pp. 1232-1238, 2020.

[45] M. Cascella, M. Rajnik, A. Aleem, S. C. Dulebohn, and R. Di Napoli, "Features, evaluation, and treatment of coronavirus (COVID-19)," in StatPearls, StatPearls Publishing, Treasure Island, FL, USA, 2021.

[46] A. Grifoni, J. Sidney, Y. Zhang, R. H. Scheuermann, B. Peters, and A. Sette, "A sequence homology and bioinformatic approach can predict candidate targets for immune responses to SARS-CoV-2," Cell Host and Microbe, vol. 27, no. 4, pp. 671.e2-680.e2, 2020.

[47] A. K. Shanker, D. Bhanu, A. Alluri, and S. Gupta, "Wholegenome sequence analysis and homology modelling of the main protease and non-structural protein 3 of SARS-CoV-2 reveal an aza-peptide and a lead inhibitor with possible antiviral properties," New Journal of Chemistry, vol. 44, no. 22, pp. 9202-9212, 2020.

[48] W. K. Oh, "COVID-19 infection in cancer patients: early observations and unanswered questions," Annals of Oncology, vol. 31, no. 7, pp. 838-839, 2020.

[49] B. Zhang, X. Zhou, C. Zhu et al., "Immune phenotyping based on the neutrophil-to-lymphocyte ratio and IgG level predicts disease severity and outcome for patients with COVID-19," Frontiers in Molecular Biosciences, vol. 7, p. 157, 2020.

[50] X. Zhang, K. Song, F. Tong et al., "First case of COVID-19 in a patient with multiple myeloma successfully treated with tocilizumab," Blood Advances, vol. 4, no. 7, pp. 1307-1310, 2020.

[51] E. J. Emanuel, G. Persad, R. Upshur et al., "Fair allocation of scarce medical resources in the time of covid-19," New England Journal of Medicine, vol. 382, no. 21, pp. 2049-2055, 2020.

[52] M. L. Ranney, V. Griffeth, and A. K. Jha, "Critical supply shortages-the need for ventilators and personal protective equipment during the covid-19 pandemic," New England Journal of Medicine, vol. 382, no. 18, 2020.

[53] M. Bersanelli, "Controversies about COVID-19 and anticancer treatment with immune checkpoint inhibitors," Immunotherapy, vol. 12, no. 5, pp. 269-273, 2020.

[54] M. Ueda, R. Martins, P. C. Hendrie et al., "Managing cancer care during the COVID-19 pandemic: agility and collaboration toward a common goal," JNCCN Journal of the 
National Comprehensive Cancer Network, vol. 18, no. 4, 2020.

[55] D. Ren, Y. Hua, B. Yu et al., "Predictive biomarkers and mechanisms underlying resistance to PD1/PD-L1 blockade cancer immunotherapy," Molecular Cancer, vol. 19, no. 1, p. 19, 2020.

[56] Y. Jin, H. Yang, W. Ji et al., "Virology, epidemiology, pathogenesis, and control of covid-19," Viruses, vol. 12, no. 4, p. 372, 2020.

[57] P. Mehta, D. F. McAuley, M. Brown, E. Sanchez, R. S. Tattersall, and J. J. Manson, "COVID-19: consider cytokine storm syndromes and immunosuppression," in The Lancet, vol. 395, no. 10229, pp. 1033-1034, 2020.

[58] F. Zhou, T. Yu, R. Du et al., "Clinical course and risk factors for mortality of adult inpatients with COVID-19 in Wuhan, China: a retrospective cohort study," The Lancet, vol. 395, no. 10229, pp. 1054-1062, 2020.

[59] J. K. Hwang, T. Zhang, A. Z. Wang, and Z. Li, "COVID-19 vaccines for patients with cancer: benefits likely outweigh risks," Journal of Hematology and Oncology, vol. 14, no. 1, 2021.

[60] Q. Ye, B. Wang, and J. Mao, "The pathogenesis and treatment of the "Cytokine Storm" in COVID-19," Journal of Infection, vol. 80, no. 6, pp. 607-613, 2020.

[61] S. Ganatra, S. P. Hammond, and A. Nohria, "The novel coronavirus disease (COVID-19) threat for patients with cardiovascular disease and cancer," in Journal of the American College of Cardiology: CardioOncology, vol. 2, no. 2, pp. 350-355, 2020.

[62] A. Shankar, D. Saini, S. Roy et al., "Cancer care delivery challenges amidst coronavirus disease -19 (covid-19) outbreak: specific precautions for cancer patients and cancer care providers to prevent spread," Asian Pacific Journal of Cancer Prevention, vol. 21, no. 3, pp. 569-573, 2020.

[63] P. Zhai, Y. Ding, X. Wu, J. Long, Y. Zhong, and Y. Li, "The epidemiology, diagnosis and treatment of COVID-19," International Journal of Antimicrobial Agents, vol. 55, no. 5, 2020.

[64] S. Lei, F. Jiang, W. Su et al., "Clinical characteristics and outcomes of patients undergoing surgeries during the incubation period of COVID-19 infection," EClinicalMedicine, vol. 21, 2020.

[65] J. Van de Haar, L. R. Hoes, C. E. Coles et al., "Author Correction: caring for patients with cancer in the COVID-19 era," Nature Medicine, vol. 26, no. 7, p. 1146, 2020a.

[66] R. E. Jordan, P. Adab, and K. K. Cheng, "Covid-19: risk factors for severe disease and death," BMJ, vol. 368, 2020.

[67] I. Kim, J. Lee, J. Lee, E. Shin, C. Chu, and S. K. Lee, "KCDC risk assessments on the initial phase of the COViD-19 outbreak in Korea," Osong Public Health and Research Perspectives, vol. 11, no. 2, pp. 67-73, 2020.

[68] W. McKibbin and R. Fernando, "The global macroeconomic impacts of covid-19: seven scenarios," Asian Economic Papers, vol. 20, no. 2, pp. 1-30, 2021.

[69] C. D. Lehman, L. R. Lamb, and H. A. D’Alessandro, "Mitigating the impact of coronavirus disease (COVID-19) vaccinations on patients undergoing breast imaging examinations: a pragmatic approach," American Journal of Roentgenology, vol. 217, no. 3, pp. 584-586, 2021.

[70] B. Robson, "COVID-19 Coronavirus spike protein analysis for synthetic vaccines, a peptidomimetic antagonist, and therapeutic drugs, and analysis of a proposed achilles' heel conserved region to minimize probability of escape mutations and drug resistance," Computers in Biology and Medicine, vol. 121, 2020.

[71] L. Eaton, "Covid-19: WHO warns against "vaccine nationalism" or face further virus mutations," BMJ, vol. 372, 2021.
[72] M. C. Mills and D. Salisbury, "The challenges of distributing COVID-19 vaccinations," EClinicalMedicine, vol. 31, 2021.

[73] A. A. M. Van der Veldt, S. F. Oosting, A. M. C. Dingemans et al., "COVID-19 vaccination: the VOICE for patients with cancer," Nature Medicine, vol. 27, no. 4, pp. 568-569, 2021.

[74] D. Calina, A. O. Docea, D. Petrakis et al., "Towards effective COVID-19 vaccines: updates, perspectives and challenges (Review)," International Journal of Molecular Medicine, vol. 46, no. 1, pp. 3-16, 2020.

[75] R. H. Dodd, K. Pickles, B. Nickel et al., "Concerns and motivations about COVID-19 vaccination," in The Lancet Infectious Diseases, vol. 21, no. 2, pp. 161-163, 2021.

[76] H. Moraliyage, D. De Silva, W. Ranasinghe et al., "Cancer in lockdown: impact of the COVID-19 pandemic on patients with cancer," The Oncologist, vol. 26, no. 2, pp. e342-e344, 2021.

[77] H. O. Al-Shamsi, W. Alhazzani, A. Alhuraiji et al., "A practical approach to the management of cancer patients during the novel coronavirus disease 2019 ( COVID-19) pandemic: an international collaborative group," The Oncologist, vol. 25, no. 6, pp. e936-e945, 2020, https://doi.org/10.1634/theoncologist.2020-0213.

[78] B. Anthony Jnr, "Implications of telehealth and digital care solutions during COVID-19 pandemic: a qualitative literature review," in Informatics for Health and Social Care, vol. 46, no. 1, pp. 68-83, 2021.

[79] M. M. Shirke, S. A. Shaikh, and A. Harky, "Tele-oncology in the COVID-19 era: the way forward?" in Trends in Cancer, vol. 6, no. 7, pp. 547-549, 2020.

[80] M. Vreugdenhil, C. Fong, P. Sanghera, A. Hartley, J. Dunn, and H. Mehanna, "Hypofractionated chemoradiation for head and cancer: data from the PET NECK trial," Oral Oncology, vol. 113, 2021.

[81] L. C. Mendez, H. Raziee, M. Davidson et al., "Should we embrace hypofractionated radiotherapy for cervical cancer? a technical note on management during the COVID-19 pandemic," Radiotherapy and Oncology, vol. 148, pp. 270-273, 2020.

[82] A. S. Pathania, P. Prathipati, B. A. A. Abdul et al., "COVID-19 and cancer comorbidity: therapeutic opportunities and challenges," in Theranostics, vol. 11, no. 2, pp. 731-753, 2020, https://doi.org/10.7150/thno.51471.

[83] R. A. Clark-Snow and C. Rittenberg, "Oncology nursing supportive care during the COVID-19 pandemic: reality and challenges," Supportive Care in Cancer, vol. 29, no. 5, pp. 2259-2262, 2021.

[84] R. Edge, C. Mazariego, Z. Li et al., "Psychosocial impact of COVID-19 on cancer patients, survivors, and carers in Australia: a real-time assessment of cancer support services," Supportive Care in Cancer, vol. 29, no. 9, pp. 5463-5473, 2021.

[85] J. Nakayama, S. A. El-Nashar, S. Waggoner, B. Traughber, and J. Kesterson, "Adjusting to the new reality: evaluation of early practice pattern adaptations to the COVID-19 pandemic," Gynecologic Oncology, vol. 158, no. 2, pp. 256-261, 2020.

[86] D. L. Bartlett, J. R. Howe, G. Chang et al., "Management of cancer surgery cases during the COVID-19 pandemic: considerations," in Annals of Surgical Oncology, vol. 27, no. 6, pp. 1717-1720, 2020.

[87] C. J. D. Wallis, G. Novara, L. Marandino et al., "Risks from deferring treatment for genitourinary cancers: a collaborative review to aid triage and management during the COVID-19 pandemic[formula presented]," in European Urology, vol. 78, no. 1, pp. 29-42, 2020.

[88] A. R. Jazieh, H. Akbulut, G. Curigliano et al., "1678P_PR the impact of COVID-19 pandemic on cancer care: a global collaborative study," Annals of Oncology, vol. 31, no. 4, pp. S1209-S1210, 2020. 